\title{
Active Piezoelectric Vibration Isolation System of Machine Tools
}

\author{
Miaoxian Guo ${ }^{1, *}$, Beizhi $\mathrm{Li}^{2}$, Jianguo Yang ${ }^{3}, \mathrm{Wei} \mathrm{Li}^{4}$ and Steven Y. Liang ${ }^{5}$ \\ ${ }^{1,2,3,4}$ Advanced Manufacturing Center of Donghua University, Shanghai, China \\ ${ }^{5}$ Georgia Institute of Technology, Atlanta, USA \\ ${ }^{*}$ Corresponding author
}

\begin{abstract}
Isolating the ultra-precision machine from vibrating base is importance in engineering fields. And the vibration isolation system is applied to reduce transmission of vibratory forces to the machine or the foundation. With the development of the vibration control techniques and force actuator in industry, active vibration isolation provides very high vibration isolation performance. In this paper, the theory and practice of vibration isolation reducing vibration transmitted from vibrating base to sensitive equipment have been studied in the paper. And a Zdirection isolation system is designed and provided by using proportional-integral-derivative controller and piezoelectric element. The approach is validated using experimental data. Results show that this vibration cancellation system can get good performance to reduce the effect of unwanted vibration and disturbances.
\end{abstract}

Keywords-active conrol; vibration isolation; machine tool; PID

\section{INTRODUCTION}

Vibration of machining process and excessive floor has detrimental effects on part quality, tool life, and productivity, so the machine needs to be isolated against disturbance mechanisms which disturb the metrology loop besides exciting structural resonances on the machine. The ability to isolate a machine from the undesirable effects of vibration has wide application. Normally there are two types of vibration isolation: passive isolation mount and active vibration isolation system. The traditional passive vibration isolation systems are based on the linear harmonic oscillator and the avoidance of resonance [1]. Additionally, the active control system cancels vibration in real time by sensing floor vibration, then expanding and contracting actuators to filter out floor motion. Generally the best isolation performance is achieved by using an active system in combination with a passive mount [2].

As passive isolation system, the knowledge has been well researched and established during last several decades. Subrahmanyan et al. [3] casts the vibration isolation problem as a control paradigm and relates the effect of the contradictory disturbance forces to sensitivity and complementary sensitivity functions. The research also describes the application of Kalman filtering techniques to the passive mount synthesis problem. Chinedum et al. [4] uses the 2D dynamics of a passively isolated system to show that coupling the vibration modes of the isolated system by judiciously locating its isolators more often than not provides conditions that lead to the reduction of residual vibrations compared to the recommended practice of decoupling. The proposed method of reducing residual vibrations is very attractive.
It is a problem that should to make the mount as stiff as possible to better support the equipment and as soft as possible to better isolate the equipment. Therefore, the active vibration isolation system is a better way to garreteer the precision machining. It provides very high vibration isolation performance without introducing a low-stiffness isolation system (such as traditional air isolators).

Chunchuan et al. [5] presents some novel results about analysis and design of low-frequency or broadband-frequency vibration isolation using a hybrid lever-type isolation system with an X-shape supporting structure in passive or semi-active control manners. Farshidianfar [2] designed an active vibration isolation system using the $\mathrm{H} \infty$ controller based on the solvability of PSO algorithm in optimization problem. Active control based on static output feedback and $\mathrm{H} \infty$ criterion was presented as a cost function and optimizing is performed by using PSO algorithm, and was validated effective reduction the resonance responses. Law's research [6] reports on a novel electro-hydraulic actuator that attenuates and isolates ground motion to keep dynamic excitations transmitted to machine tools below permissible levels. The actuator is designed with a high passive stiffness to support inertial loads of the machine.

Based on the previous research, this paper implements an active vibration isolation system for machine tool based on piezoelectric ceramics actuators and dynamic acceleration sensors. In this system, the base of the machine tool in Zdirection is driven by a specially designed active stage to control the relative vibration between the stage and the ground. PID control algorithm is used, and the system is designed and tests. This work also shows the important of the vibration isolation system in the machining system.

\section{ACtiVE VibRATION CANCELLATION ANALYSIS}

\section{A. Model Description}

Figure I shows the active vibration isolation system, it cancels vibration in real time by sensing ground vibration, then expanding and contracting piezoelectric actuators to filter out floor motion.

Consider the single-axis isolator connecting the ground to a machine tool $\mathrm{m}$, the system consists of a spring $k$, a damping $c$ in parallel with a force actuator $F a$; the objective is to isolate the machine $M$ from the vibration motion $x_{g}$ of ground. The governing equations are

$$
m \ddot{x}_{m}+c\left(\dot{x}_{m}-\dot{x}_{g}\right)+k\left(x_{m}-x_{g}\right)=F_{a}
$$




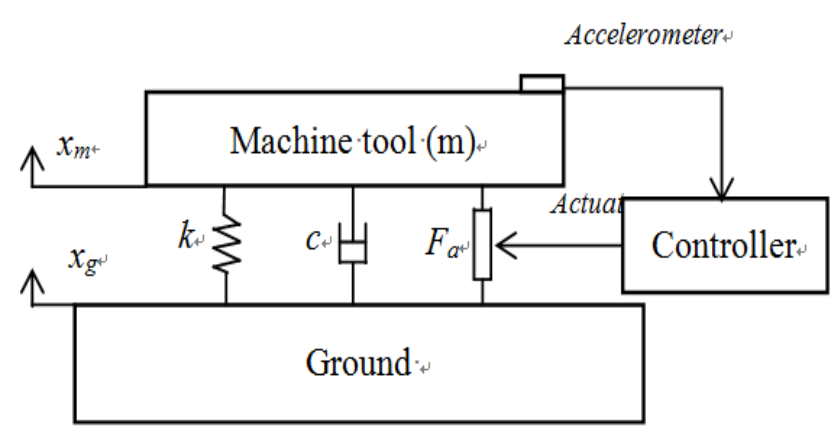

FIGURE I. SINGLE-AXIS ACTIVE ISOLATOR

The expression describes that the response is consists of the response to the disturbance load and the payload response to the actuator. If an accelerometer or a geophone is attached to the machine payload, measuring the absolute acceleration, the transmissibility is

$$
G(s)=\frac{x_{m}}{x_{g}}=\frac{c s+k}{m s^{2}+c s+k}
$$

\section{B. PID Control Algorithm}

PID controller in the paper continuously calculates an error value as the difference between a measured vibration variable and a desired set point. The controller attempts to minimize the vibration on the machine by adjustment of a control variable, to a new value determined by a weighted sum:

$$
\begin{aligned}
& u(t)=K_{p}\left[e(t)+\frac{1}{T_{i}} \int_{0}^{t} e(t) d t+\frac{T_{d} d e(t)}{d t}\right] \\
& =K_{p} e(t)+K_{i} \int_{0}^{t} e(t) d t+K_{d} \frac{d e(t)}{d t}
\end{aligned}
$$

where $K_{p}, K_{i}$, and $K_{d}$ are non-negative coefficients for the proportional, integral and derivative terms respectively. In this model, $\mathrm{P}$ accounts for present values of the vibration signal error, I accounts for past values of the error, and D accounts for possible future values of the error, based on its current rate of change [7]. When the objective function $r(t)$ is set as zero, then using the machine tool vibration acceleration $\ddot{x}_{m}$ as process variable $\mathrm{y}(\mathrm{t})$, the error

$$
e(t)=r(t)-y(t)=-\ddot{x}_{m}
$$

And

$$
F_{a}=-K_{p} \ddot{x}_{m}-K_{i} \int_{0}^{t} \ddot{x}_{m} d t-K_{d} \frac{d \ddot{x}_{m}}{d t}
$$

Calculate the equation (1) and (5),

$K_{d} \frac{d \ddot{x}_{m}}{d t}+\left(m+K_{p}\right) \ddot{x}_{m}+\left(c+K_{i}\right) \dot{x}_{m}+K_{i}=\left(c+K_{i}\right) \dot{x}_{g}+K_{i}$

And then it come the following function by applying Laplace transform:
$G(s)=\frac{\left(c+K_{i}\right) s+K_{i}}{K_{d} s^{3}+\left(m+K_{p}\right) s^{2}+\left(c+K_{i}\right) s+K_{i}}$

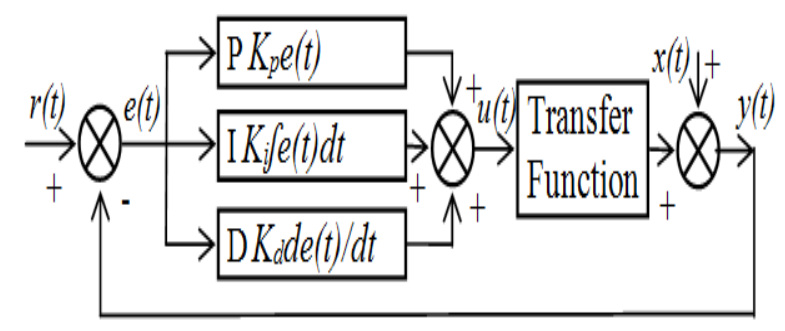

FIGURE II. A BLOCK DIAGRAM OF A PID CONTROLLER

\section{DESIGN OF THE SYSTEM}

\section{A. Experimental}

As shown in the Figure III, the experimental system consists of three parts, machine tool system, vibration excitation system and active vibration isolation system.
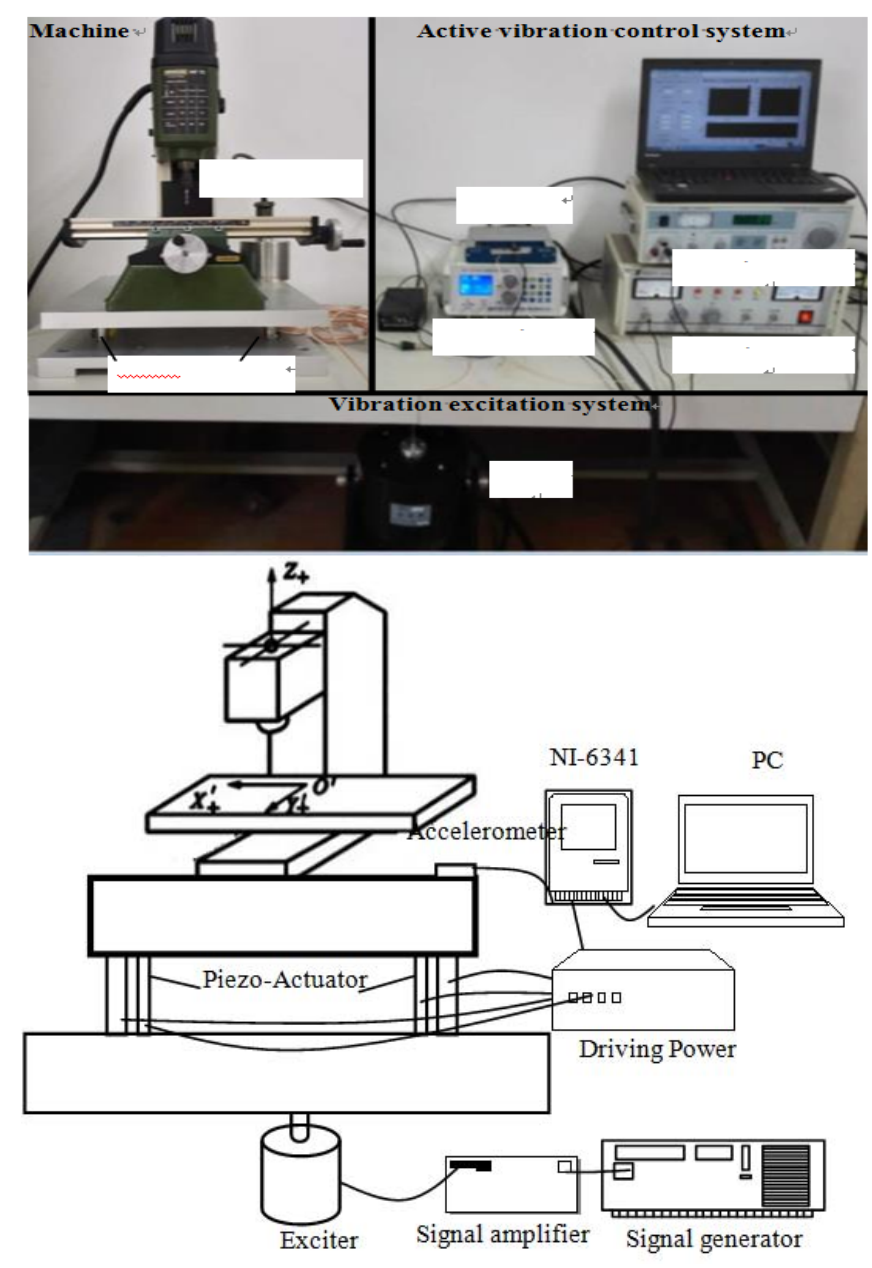

FIGURE III. EXPERIMENTAL SETUP

In the machine tool system, the accelerometers monitor the vibration and the piezoelectric ceramics actuator cancels 
vibration in real time; in the vibration excitation system, the exciter general the vibration to simulate the Z-direction disturbance vibration from the foundation; and in the control system, the NI data acquisition 6341 acquire the vibration signal by accelerometer and output control signal to diving power device using the PC program. The data acquisition and PID control algorithm program are developed by the LabVIEW.

\section{B. Calibration of the Piezoelectric Actuator}

In order to control the piezoelectric ceramics actuator by voltage signal, the displacement characteristic should be obtained in the paper. As shown in the Figure IV, the TESATRONIC TT20 instrument and TESA GT21 inductance measuring head is applied to measure the displacement amplitude by the driving of different voltage. The diving power device is set to manual mode, and the calibration is performed by varying the input voltage from 0 to $150 \mathrm{~V}$ in both the ascending and descending.

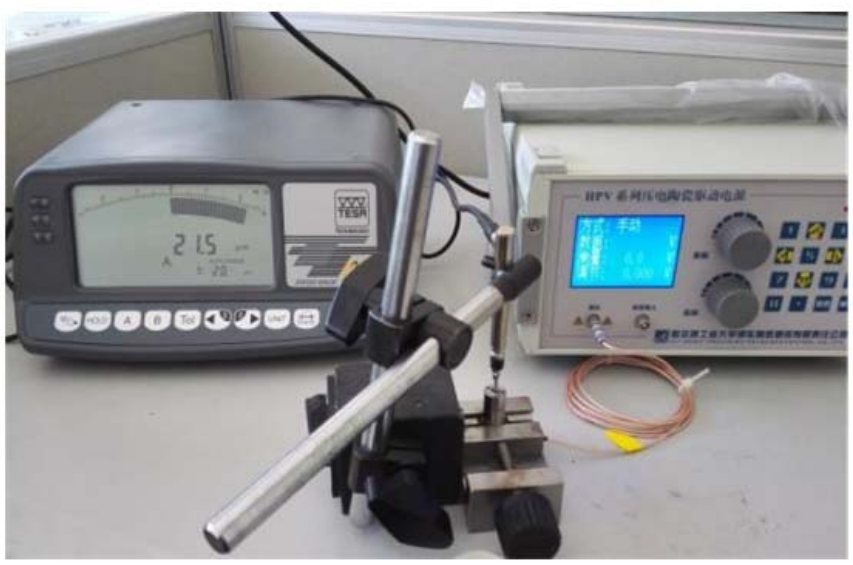

FIGURE IV. CALIBRATION SYSTEM

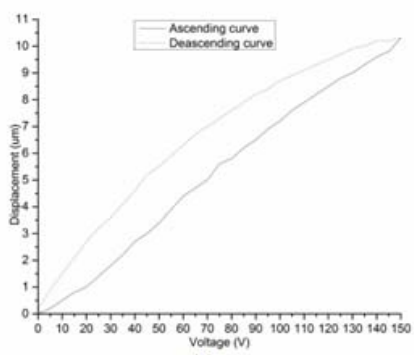

\#1
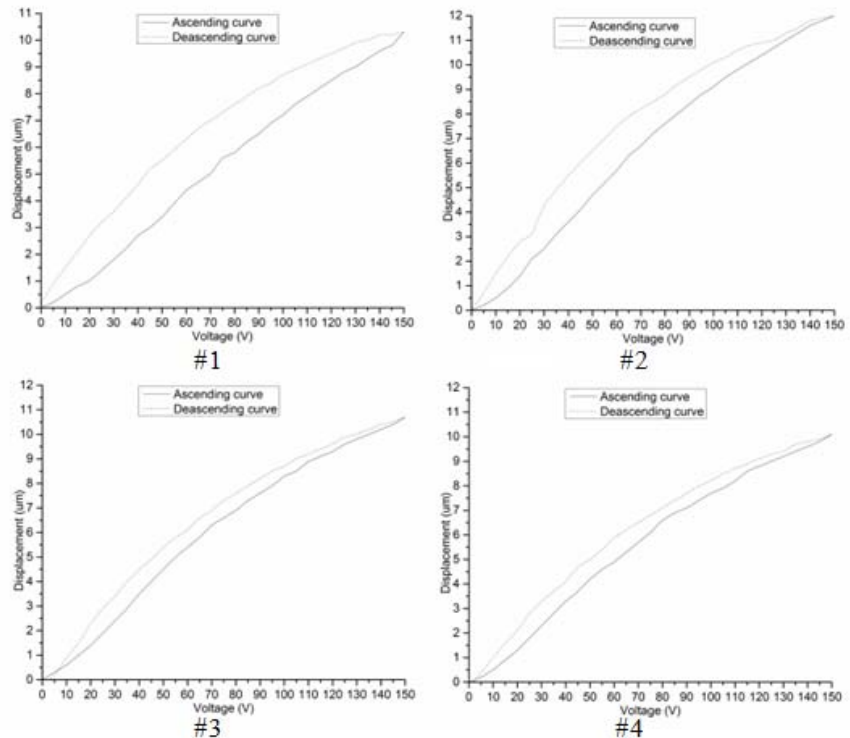

FIGURE V. DISPLACEMENT CHARACTERISTIC CURVE OF FOUR PIEZOELECTRIC CERAMICS ACTUATOR
The calibration result in Figure $\mathrm{V}$ shows that the piezoelectric ceramics bender does not follow the same path to return its original position. It is observed that piezoelectric actuator has a deflection error (hysteresis).

\section{Experimental Parameters}

Signature testing was carried out with the isolation system in the paper. First the harmonic disturbance at a certain frequency of $10 \mathrm{~Hz}$ or $20 \mathrm{~Hz}$ is applied at the exciter, and then the scan mode of $5 \mathrm{~Hz}-100 \mathrm{~Hz}$ is used in the experimental system to further verify the vibration isolation result.

\section{RESULTS AND DisCUSSIONS}

It is important to ensure that acceleration of machine and structure are minimized as far as possible. The experimental test runs begin with active control off. And then, the control is turned on. The excitation was at $10 \mathrm{~Hz}, 20 \mathrm{~Hz}$ and $10-100 \mathrm{~Hz}$ scan in the case, respectively.

The experimental results for the system are depicted in Figure VI-VIII. These diagrams indicate the effects of disturbance reduction by using active control system. They show a comparison of control system performance: the vibration amplitude of system is isolated when the control system is on, the reduction of relative vibration along the Zdirection is about $40 \%$ in the case of control on. Therefore, the active vibration isolation system has good isolation performance against the disturbance acting on the system within the frequency range.

In the study, the vibration isolation theoretical model of piezoelectric actuator is established. Also, the control theory and PID algorithms for active vibration isolation are explored. Then, the hardware experiment platform for active vibration isolation control system is built. The vibration exciter provide primary excitation signal for this hardware platform. NI system collects primary and controlled vibration signal, and then outputs the control voltage. Both the signature recording and control signal are done with the help of LabVIEW software. Vibration isolation test is conducted at last to verify the validity of the system.

Furthermore, no matter what the excitation signal is, a significant vibration reduction signal can be obtained, which verifies the validity of the active vibration isolation system. This investigation will provide an important theoretical foundation for the active vibration isolation system of machine tools and other precision equipment.

\section{ACKNOWLEDGMENT}

This project is supported by National 863 High Technology R\&D Program (No. 2012AA041309). 


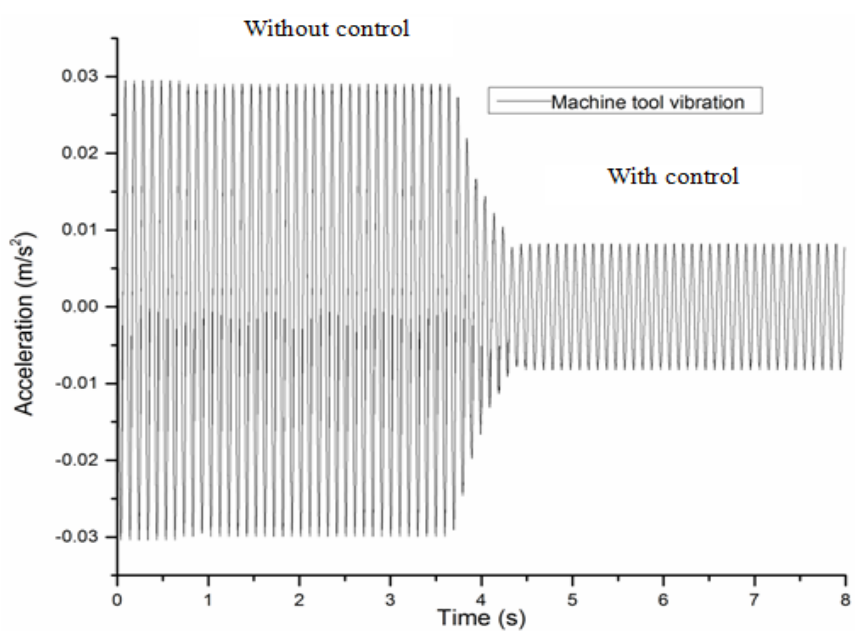

FIGURE VI. COMPARISON OF MACHINE TOOL VIBRATION WITH AND WITHOUT CONTROL (10HZ EXCITATION)

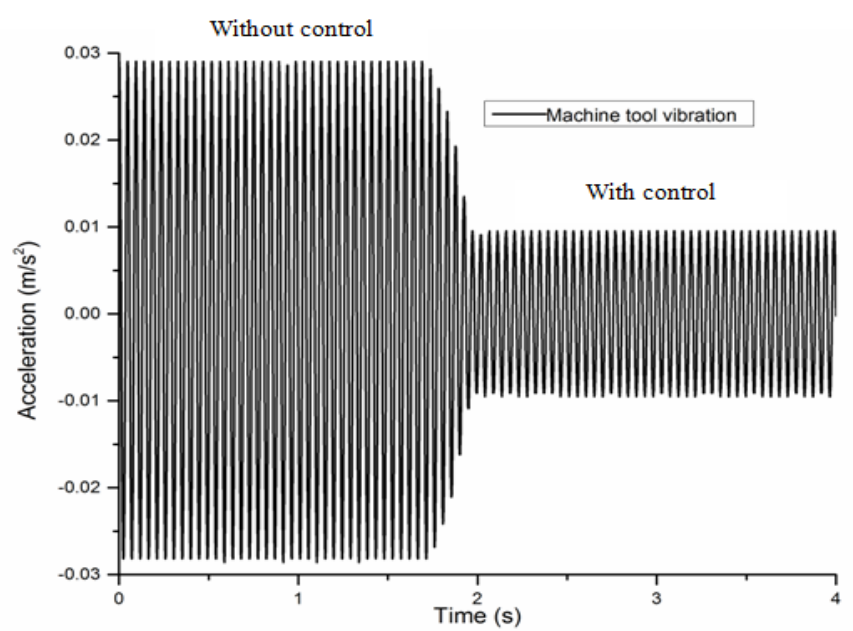

FIGURE VII. COMPARISON OF MACHINE TOOL VIBRATION WITH AND WITHOUT CONTROL (20HZ EXCITATION)

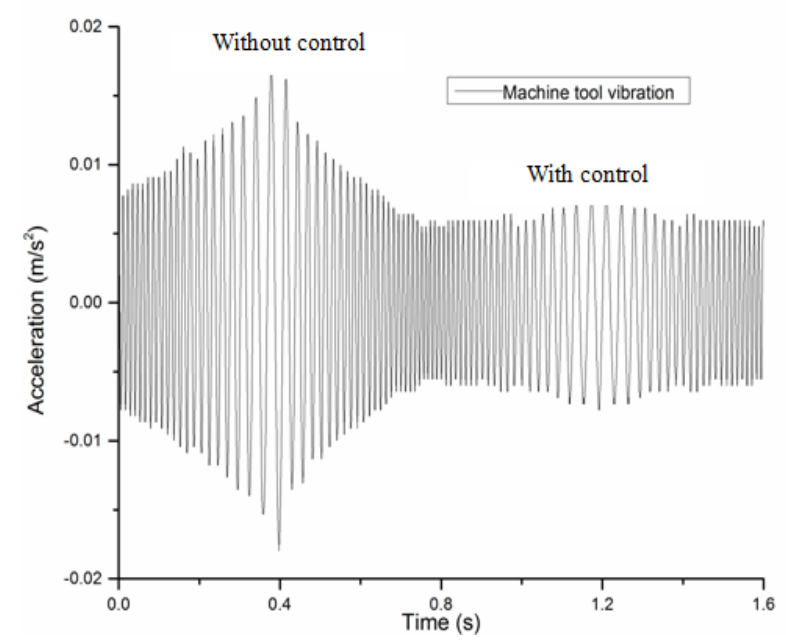

FIGURE VIII. COMPARISON OF MACHINE TOOL VIBRATION WITH AND WITHOUT CONTROL (10-100HZ BAND EXCITATION)

\section{REFERENCES}

[1] Virgin, L., Vibration Isolation, D. Wagg and L. Virgin, D. Wagg and L. Virgin Editors. 2012, Springer Vienna. p. 111-134.

[2] Farshidianfar, A., et al., Active vibration iso-lation of machinery and sensitive equipment using $\mathrm{H} \infty$ control criterion and particle swarm optimization method. Meccanica, 2012. 47(2): p. 437-453.

[3] P.K. Subrahmanyan, D.L. Trumper, Synthesis of passive vibration isolation mounts for machine tools: A control system paradigm. Proceedings of the American Control Conference, Chicago, June 2000: 2886-2891.

[4] Chinedum E. Okwudire, Jihyun Lee, Minimi-zation of the residual vibrations of ultra-precision manufacturing machines via optimal placement of vibration isolators. Precision Engineering 37 (2013) 425432

[5] Chunchuan Liu, Xingjian Jing, Fengming Li, Vibration isolation using a hybrid lever-type isolation system with an X-shape supporting structure, International Journal of Mechanical Sciences, Vol-ume 98, July 2015, Pages 169-177

[6] M. Law, M. Wabner, A. Colditz, M. Kolouch, S. Noack, S. Ihlenfeldt, Active vibration isolation of machine tools using an electro-hydraulic actuator. CIRP Journal of Manufacturing Science and Tech-nology 10 (2015) 36-48

[7] Araki M. PID control. Control Systems, Ro-botics and Autimation, 1995, 1(2): 1-23.Writer's Handbook. Mill Valley, CA: University Science, 1989. 